\title{
Milk and kefir maintain aspects of health during doxorubicin treatment in rats
}

\author{
L. K. Stewart, ${ }^{1 *} \dagger$ P. Smoak, ${ }^{1 *}$ D. S. Hydock, ${ }^{1}$ R. Hayward, ${ }^{1}$ K. O’Brien, ${ }^{2}$ J. K. Lisano, ${ }^{1}$ C. Boeneke, ${ }^{2}$ \\ M. Christensen, ${ }^{1}$ and A. Mathias ${ }^{1}$ \\ ${ }^{1}$ School of Sport and Exercise Science and the University of Northern Colorado Cancer Rehabilitation Institute, University of Northern Colorado, \\ Greeley 80639 \\ ${ }^{2}$ School of Nutrition and Food Sciences, Louisiana State University Agricultural Center, Baton Rouge 70803
}

\section{ABSTRACT}

Doxorubicin (DOX), a powerful anthracycline antibiotic commonly used to treat a wide variety of cancers, is associated with the production of reactive oxygen species that cause oxidative damage, resulting in cardiac dysfunction. Components of dairy may have protective effects against DOX-induced cardiac damage. Kefir is a naturally fermented milk product containing antioxidants, probiotic bacteria, and yeast in addition to the protective components of dairy. We explored the effects of dietary milk and kefir on DOX-induced cardiotoxicity in rats. We used singly housed, 10-wk-old male Sprague Dawley rats assigned to 1 of 3 isocaloric diets, control $(\mathrm{CON} \mathrm{n}=24)$, milk $(\mathrm{MLK}, \mathrm{n}=24)$, or kefir $(\mathrm{KEF}, \mathrm{n}=24)$, with equivalent macronutrient profiles. After a 9-wk dietary intervention, all animals were given either a bolus injection $(15 \mathrm{mg} / \mathrm{kg})$ of DOX (CON-DOX $\mathrm{n}=12$; MLK-DOX $\mathrm{n}=12$, KEF-DOX $\mathrm{n}=12$ ) or saline (CON-SAL $\mathrm{n}=12$; MLK-SAL $\mathrm{n}$ $=12$; KEF-SAL $\mathrm{n}=12$ ). Body weight, grip strength, echocardiographic evaluation of cardiac geometry, and cardiac function were evaluated using echocardiography at $5 \mathrm{~d}$ postinjection and data were analyzed using ANOVA. Survival at d 5 post-DOX injection was 92 and $100 \%$ in KEF-DOX and MLK-DOX, respectively, and $75 \%$ in CON-DOX. By the last week of the dietary intervention, and just before injection with saline or DOX, CON weighed significantly (14\%) more than the MLK and KEF. The DOX treatment resulted in significant reductions in body weight; however, we found no diet $x$ drug interactions. The DOX treatment reduced peak grip strength compared with SAL; when compared with pre-injection measures, MLK-DOX rats did not experience a significant reduction in peak grip strength compared with CON-DOX and SAL-DOX

Received August 20, 2018.

Accepted November 20, 2018.

*These authors contributed equally to this work.

†Corresponding author: Laura.Stewart@unco.edu rats. Heart mass in MLK and KEF was significantly higher when compared with CON. In summary, 9 wk of milk or kefir ingestion resulted in lower body size and higher heart mass after DOX treatment. Additionally, MLK preserved peak grip strength after DOX treatment, whereas KEF or CON did not. We observed no consistent protective effects with respect to heart dimensions and function. These findings suggest that long-term milk or kefir ingestion may be helpful in optimizing health before and during doxorubicin treatment. Key words: dairy, chemotherapy, cancer

\section{INTRODUCTION}

Cancer is one of the most prevalent diseases in humans today. Approximately $39.6 \%$ of men and women will be diagnosed with cancer at some point during their lifetime. Cancer treatment is becoming more effective, and the number of individuals living beyond cancer diagnosis has increased each year. By the year 2024, there will be approximately 19 million cancer survivors in the United States (ACS, 2017). Doxorubicin, an anthracycline antibiotic, is widely used as treatment for many cancers. Doxorubicin acts as an antitumor agent by intercalating DNA, thereby reducing cancer cell replication (Yang et al., 2014); however, doxorubicin can also alter the health of other tissues when used as a chemotherapy drug, resulting in irreversible heart damage, immune function dysfunction, and disruption of the intestinal microbiome (Pugazhendhi et al., 2018). In fact, recent studies using mice suggested that harmful changes in the myocardium can occur in as little as 2 wk after receiving what is considered a safe dose of $3 \mathrm{mg} / \mathrm{kg}$ per week of doxorubicin (Desai et al., 2013; Piotrowska et al., 2017). The development of acute doxorubicin cardiotoxicity occurs in approximately $11 \%$ of patients and has the potential to be reversible; however, chronic doxorubicin cardiotoxicity, although much less common, is associated with a significant increase in mortality (Chatterjee et al., 2010).

Although pharmacological treatments aimed at reducing doxorubicin-induced side effects, such as 
dexrazoxane, are used clinically, survivors often have to endure additional immunosuppressive side effects (Langer, 2014). Consequently, the incorporation of foods including dairy products presents an attractive intervention option. In fact, whey protein is protective against doxorubicin-induced cardiotoxicity (Alexieva et al., 2014). The probiotic component of many dairy foods presents a unique opportunity to mitigate doxorubicininduced side effects. Recent animal studies show that consumption of probiotics may have powerful anticytotoxic effects (Sharma et al., 2011), and that a diet with a yogurt component can improve membrane integrity and cardiac contractility in a rat model of doxorubicininduced cardiomyopathy (Abu-Elsaad et al., 2015).

Despite these promising results, many cancer survivors develop a sensitivity to lactose and avoid dairy products during and after treatment (Osterlund et al., 2004). Kefir is a fermented milk product that contains a mixed microbial culture of lactic acid bacteria and yeasts. Kefir traditionally contains significantly less lactose than milk, and regular kefir consumption has been shown to improve lactose digestion and tolerance in adults (Hertzler and Clancy, 2003). Although this beverage has been used extensively in the Middle East, Russia, and eastern Europe for thousands of years (Farnworth, 2015), exploration of the health benefits of kefir today is scarce (Ahmed et al., 2013). In addition to the health effects derived directly from bacteria and probiotics, kefir also contains naturally occurring metabolic by-products of bacteria and yeast fermentation, including biologically active peptides, exopolysaccharides, and organic acids that are also thought to play important roles in the protective effects and immune system regulation (Garofalo et al., 2015). To date, no study has explored the effects of kefir on doxorubicininduced side effects.

Given the significant adverse side effects associated with doxorubicin and the potential health benefits of milk and kefir, the use of milk or kefir before and during doxorubicin treatment may be an attractive dietary intervention for individuals anticipating or receiving cancer treatment. Consequently, the purpose of our study was to explore whether daily ingestion of milk or kefir would help improve survival and protect against doxorubicin-induced changes in BW, grip strength, and in vivo cardiac muscle size and function.

\section{MATERIALS AND METHODS}

\section{Animals and Diets}

Male, 10-wk-old Sprague Dawley rats ( $\mathrm{n}=72$; Envigo, Indianapolis, IN) were assigned to 1 of 3 diet groups and given one of the following liquid rodent diets for 9 wk: standard rodent liquid diet (CON; Teklad, Harland, Madison WI); standard rodent liquid diet and reduced-fat milk (MLK); or standard rodent diet and reduced-fat kefir (KEF). It is important to note that at the beginning of the study, we randomized rats into 2 groups (MLK and KEF). After obtaining additional funds and after the kefir and milk dietary interventions were underway, the third, nondairy, liquid control diet group was incorporated into the study. The CON rats were purchased from the same company and were exactly the same age at the start of the dietary intervention. It is important to note that all CON animals were not part of the randomization of the animals to KEF and MLK groups. All animals were single housed in an environmentally controlled facility on a 12:12 photoperiod cycle with food and water provided ad libitum. Diets were isocaloric and contained the same macroand micronutrient content (Table 1). Low-fat cow milk was obtained from Whole Foods (Baton Rouge, LA). Kefir was produced at the Louisiana State University Creamery using low-fat, pasteurized, nonhomogenized cow milk (Whole Foods) that was fermented in gallonsized, lidded glass containers by inoculation with $30 \mathrm{~g}$ of kefir grains (Cultures for Health, Sioux Falls, SD) per $3.78 \mathrm{~L}$ (1 gallon) of milk. The kefir grains were contained in unbleached cotton tea bags, which were sterilized in boiling water before addition of the kefir grains. The milk was allowed to ferment at $25^{\circ} \mathrm{C}$ for approximately $24 \mathrm{~h}$ or until a $\mathrm{pH}$ of 4.6 was obtained. The milk and kefir were then placed in refrigerated storage and shipped on ice, overnight to the University of Northern Colorado. The control, milk, and kefir liquid diets were prepared daily at the University of Northern Colorado and provided to the animals every $12 \mathrm{~h}$ via sipper tube. All procedures were approved by the Institutional Animal Care and Use Committee.

Table 1. Nutrient composition of complete liquid $\operatorname{diets}^{1}$

\begin{tabular}{lrrr}
\hline Item & CON & \multicolumn{1}{c}{ MLK } & \multicolumn{1}{c}{ KEF } \\
\hline Energy (kcal) & 999.6 & $1,001.0$ & $1,001.0$ \\
Protein (g) & 45.1 & 45.2 & 45.2 \\
Carbohydrates (g) & 146.6 & 147.1 & 147.1 \\
Lactose (g) & 0.0 & 28.8 & 28.8 \\
Fat (g) & 26.0 & 26.0 & 26.0 \\
Fiber (g) & 11.5 & 11.6 & 11.6 \\
Calcium (g) & 1.3 & 1.3 & 1.3 \\
Phosphorus (g) & 0.8 & 0.8 & 0.8 \\
Potassium (g) & 0.9 & 0.9 & 0.9 \\
Sodium (g) & 0.3 & 0.3 & 0.3 \\
Magnesium (g) & 0.1 & 0.1 & 0.1 \\
Vitamin A (IU) & $5,414.9$ & $5,418.0$ & $5,418.0$ \\
Vitamin D (IU) & 561.8 & 570.0 & 570.0 \\
Choline (mg) & 358.5 & 358.5 & 358.5 \\
\hline
\end{tabular}

${ }^{1}$ Values are per $1,000 \mathrm{~mL}$ of control $(\mathrm{CON})$, milk (MLK), and kefir $(\mathrm{KEF})$ diets. 


\section{Doxorubicin Administration}

After the 9-wk dietary intervention period, animals received an intraperitoneal injection of either saline (SAL) or $15 \mathrm{mg} / \mathrm{kg}$ of doxorubicin (DOX), resulting in the following treatment groups: CON-SAL, CONDOX, MLK-SAL, MLK-DOX, KEF-SAL, and KEFDOX. Animals remained on their respective diets for the following $5 \mathrm{~d}$ postinjection. Animal survival was monitored throughout this period, and on d 5 postinjection we evaluated BW, grip strength, and cardiac function. Each animal was then anesthetized with an i.p. injection of heparinized sodium pentobarbital. When the tail pinch reaction was absent, the heart was excised and weighed immediately.

\section{Grip Strength Evaluation}

Skeletal muscle function in the whole animal was assessed for all animals using a grip strength meter designed for use with rats (Columbus Instruments, Columbus, $\mathrm{OH}$ ). Each rat was held over the pull bar until it grabbed the pull bar. The animal was then pulled gently and steadily in a straight horizontal line away from the meter until the animal released the pull bar. The meter then displayed the highest force applied, which was then recorded. The animal was returned to its cage for 5 min until the next test. Each animal was tested 5 times per session with the same handler, and the highest force achieved was used as the animal's peak grip strength.

\section{Cardiac Dimension and Function Evaluation}

Rats were sedated with isoflurane and in vivo cardiac function evaluation was performed using transthoracic echocardiography (Toshiba Nemio 30:10 MHz pediatric transducer; Toshiba, Tokyo, Japan). An M-mode image of the left ventricle was used to calculate left ventricular septal and posterior wall thicknesses at the end systole and diastole and left ventricular end-systolic and enddiastolic diameter. Mitral and aortic valve blood flow profiles (including maximal and mean blood flow velocities) were also obtained using pulsed wave Doppler images. Both M-mode and Doppler data were obtained from 3 consecutive cardiac cycles and averaged for each animal. All cardiac dimension and function evaluations were performed by a single researcher blinded to the treatments groups.

\section{Statistics}

A Kaplan-Meier analysis was used to evaluate survival curves. The effects of the diet and drug were explored with a 3 (diet; CON, MLK, KEF) $\times 2$ (drug; SAL, DOX) ANOVA (JMP version 12, SAS Institute Inc., Cary, NC). Changes in BW and grip strength between the preinjection and 5-d postinjection measures were identified using a 3 (diet) $\times 2$ (drug) by time (repeated measures pre- to postinjection) ANOVA (JMP). When a significant $F$-value was observed in diet, drug, and diet by drug interaction, a Tukey test was used post hoc to identify specific group differences. All results are presented as mean \pm standard deviation. Significance was set at the $\alpha=0.05$ level.

\section{RESULTS}

\section{Survival}

All SAL-injected groups (CON, MLK, and KEF) survived the 5 -d postinjection period. Survival to $5 \mathrm{~d}$ post-DOX injection was $92 \%$ (11 out of 12 animals surviving) in KEF-DOX and 100\% (12 out of 12 animals surviving) in MLK-DOX. Survival in CON-DOX at the 5 -d time point was $75 \%$ (9 out of 12 animals surviving). We found no significant differences in the survival curves (Figure 1).

\section{BW}

Average BW before the start of the dietary interventions (wk 0) was $319.94 \pm 17.70 \mathrm{~g}$ and we observed no significant differences among CON, MLK, and KEF ( $P$ $=0.38)$. By the last week of the dietary intervention, and just before injection with saline or doxorubicin (wk 9), CON (444.54 \pm 25.42 ) weighed significantly (14\%) more than the MLK (381.67 \pm 72.52$)$ and KEF

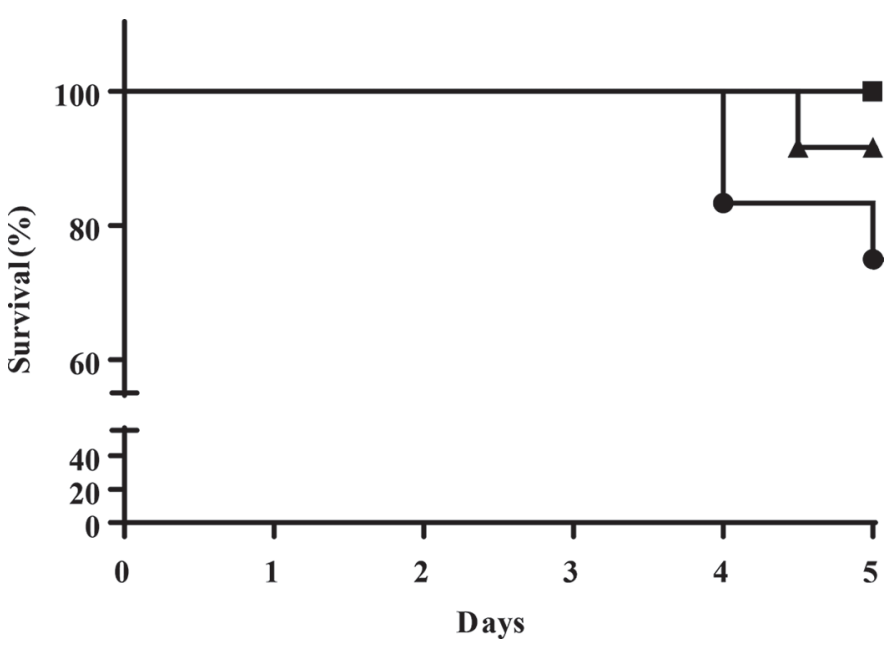

Figure 1. Percent of animals surviving the 5-d post-doxorubicin (DOX) injection period in control (CON-DOX, •), milk (MLK-DOX, $\mathbf{\square}$ ), and kefir (KEF-DOX, $\mathbf{\Delta}$ ) treatment groups. 


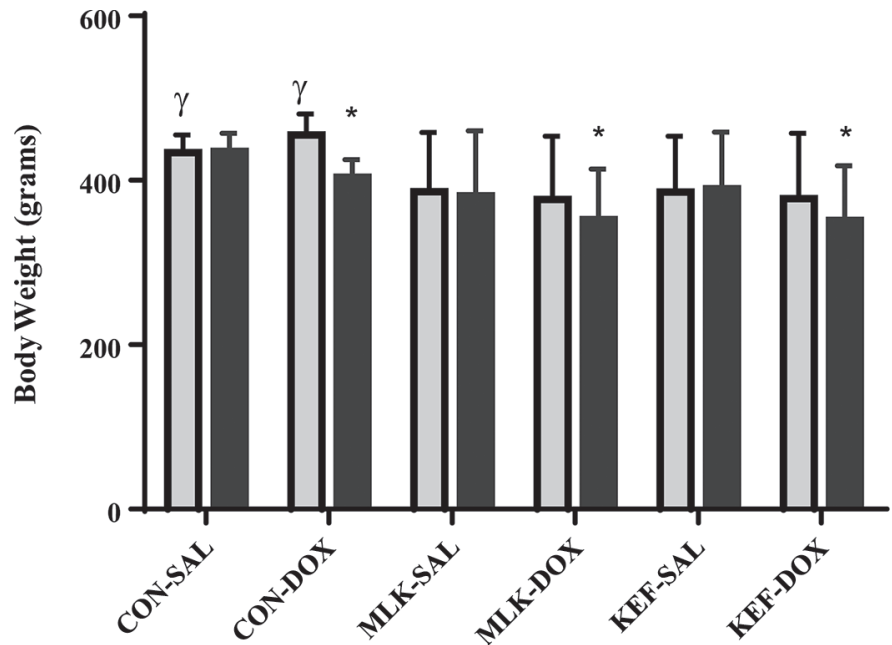

Figure 2. Mean $( \pm \mathrm{SD})$ preinjection (gray) and d-5 postinjection (black) BW for control (CON), milk (MLK), and kefir (KEF) groups injected with either saline (SAL) or doxorubicin (DOX): CON-SAL (n $=12)$, CON-DOX $(\mathrm{n}=9)$, MLK-SAL $(\mathrm{n}=12)$, MLK-DOX $(\mathrm{n}=12)$, KEF-SAL $(\mathrm{n}=12)$, and KEF-DOX $(\mathrm{n}=11)$. *Significant time $\times$ drug interaction $(P<0.0001)$, where DOX lost more BW compared with SAL. $\gamma=$ CON heavier than MLK- and KEF-treated animals before injection with either SAL or DOX $(P=0.0007)$.

$(381.65 \pm 72.82)$. A repeated-measure ANOVA of the preinjection and 5-d postinjection data revealed no diet by drug interaction. However, we noted a significant time by drug interaction, where DOX lost $8 \%$ of BW compared with SAL rats, who gained $0.6 \%$ of BW by d 5 postinjection. $(P<0.0001$; Figure 2$)$. The CONSAL $(+0.003 \%)$, KEF-SAL $(+0.01 \%)$, and MLK-SAL $(-1.2 \%)$ rats had no to small losses in BW, whereas the CON-DOX, MLK-DOX, and KEF-DOX lost an average of $11.4,6.6$, and $7.1 \%$ of BW, respectively (Figure $2)$.

\section{Grip Strength}

Animals treated with SAL had peak grip strengths $(16.2 \pm 1.6 \mathrm{~N})$ that were $11 \%$ higher than those treated with DOX $(14.4 \pm 1.9 \mathrm{~N}$; Figure 3a). When comparing preinjection to 5 -d postinjection measures, both CON-DOX $(P=0.005)$ and KEF-DOX $(P=0.0028)$ experienced significant decreases in peak grip strength, whereas we found no significant changes in MLK-DOX and all SAL-treated groups.

\section{Heart Dimensions}

Heart mass averaged $1.34 \pm 0.012 \mathrm{~g}$ in KEF and MLK and was significantly larger when compared with CON $(P<0.0001)$. The DOX rats' hearts were also $20.8 \%$ smaller than SAL $(P<0.0001)$. We observed no significant differences or interactions observed for mean septal wall thickness at systole measures; however, septal wall thickness at diastole averaged $1.73 \pm$ $0.44 \mathrm{~mm}$ and CON was $40 \%$ smaller than the MLK and $\mathrm{KEF}$ groups $(P=<0.0001)$. We also noted a significant diet and diet $\times$ drug interaction with respect to left ventricular dimension at systole (LVDs), where $\mathrm{KEF}$ was $0.10 \mathrm{~mm}$ larger when compared with CON. Interestingly, KEF-SAL LVDs was significantly (6.8\%) larger than CON-DOX, MLK-DOX, and CONSAL. Left ventricular dimension at diastole (LVDd)

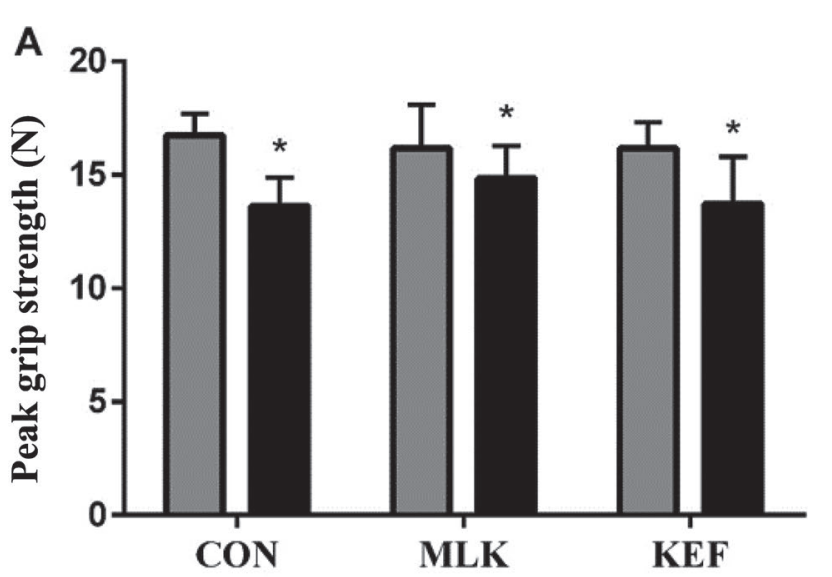

B

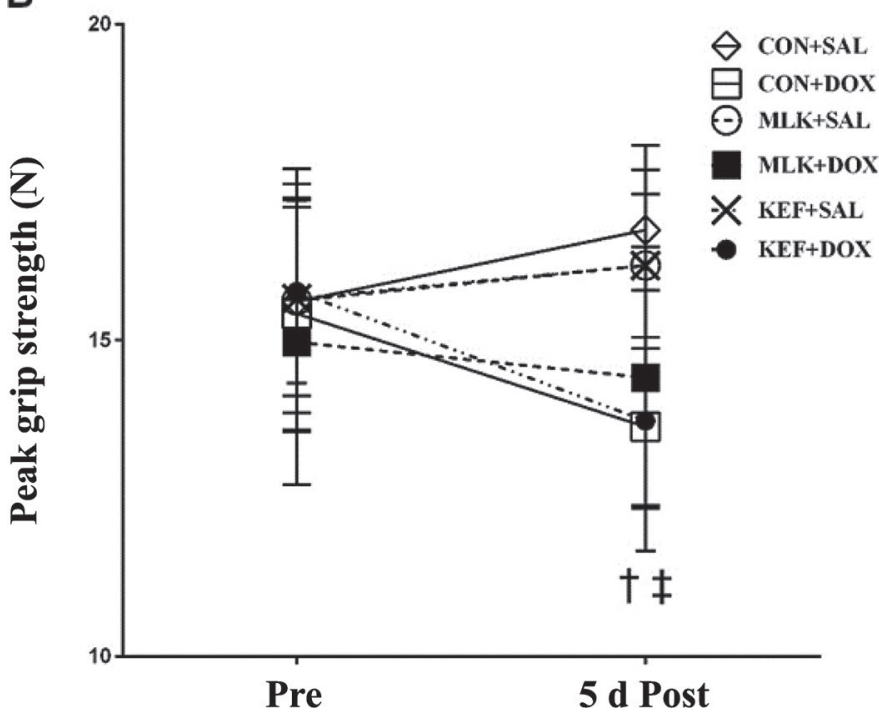

Figure 3. Mean $( \pm \mathrm{SD})$ peak grip strength measures at (a) $5 \mathrm{~d}$ postinjection and (b) preinjection and $5 \mathrm{~d}$ postinjection for control (CON), milk (MLK), and kefir (KEF) groups injected with either saline (SAL, gray) or doxorubicin (DOX, black): CON-SAL $(\mathrm{n}=12)$, CON-DOX $(\mathrm{n}=9)$, MLK-SAL $(\mathrm{n}=12)$, MLK-DOX $(\mathrm{n}=12), \mathrm{KEF}-$ SAL $(\mathrm{n}=12)$, KEF-DOX $(\mathrm{n}=11)$. ${ }^{*}$ Significant drug effect where DOX peak grip strength measures are lower than SAL $(P<0.0001)$. $\dagger$ and $\ddagger=$ significant decrease in CON-DOX $(P=0.005)$ and KEF-DOX $(P=0.0028)$ average peak grip strength measures when comparing preinjection to $5 \mathrm{~d}$ postinjection. 
Table 2. Echocardiography-derived cardiac geometry ${ }^{1,2}$

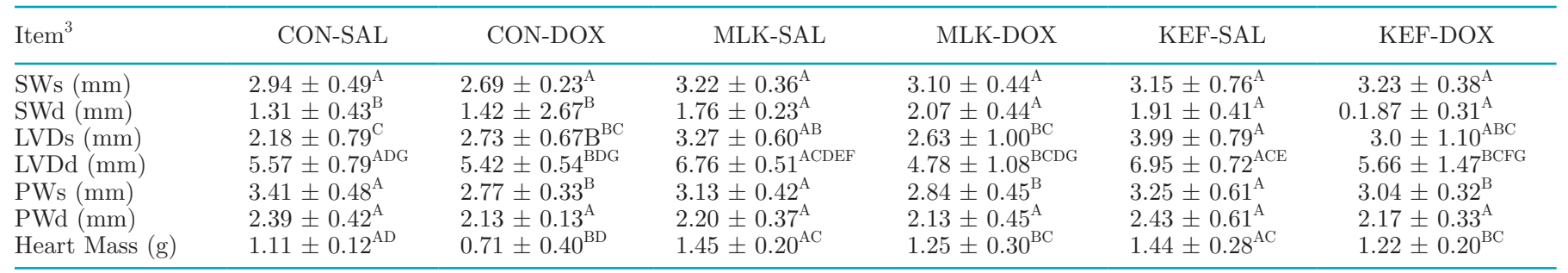

${ }^{\mathrm{A}-\mathrm{G}}$ Similar letters indicate no significant difference $(P>0.05)$ among groups within each measure.

${ }^{1}$ Data are mean $\pm \mathrm{SD}$.

${ }^{2} \mathrm{CON}=$ control; $\mathrm{MLK}=$ milk: $\mathrm{KEF}=$ kefir; $\mathrm{SAL}=$ saline; $\mathrm{DOX}=$ doxorubicin.

${ }^{3} \mathrm{SWs}=$ septal wall systole, no significant differences among groups; SWd $=$ septal wall diastole, diet effect $P<0.0001 ;$ LVDs $=$ left ventricular dimension systole, diet effect $P=0.0007$, diet $\times$ drug interaction $P=0.01 ;$ LVDd $=$ left ventricular dimension diastole, diet effect $P=0.02$, drug effect $P<0.0001$, and diet $\times$ drug interaction, $P=0.006$; PWs $=$ posterior wall systole, drug effect $P=0.0009$; PWd $=$ posterior wall diastole, no significant differences among groups; heart mass, diet effect $P<0.0001$, and drug effect $(P<0.0001)$.

was significantly influenced by $\operatorname{diet}(P=0.02)$, drug $(P<0.0001)$, and diet $\times$ drug interaction $(P=0.006)$, where KEF LVDd was $14.7 \%$ larger than CON and DOX LVDd was $21.4 \%$ smaller than SAL. We found no significant effects or interactions with respect to posterior wall at diastole; however, drug effects $(P=0.0009)$ were observed for posterior wall at systole, where DOX was $11.7 \%$ smaller than SAL (Table 2).

\section{Heart Function}

Aortic peak flow velocity was significantly (59.1\%) lower in DOX compared with CON $(P<0.0001)$, and we observed a significant diet $\times$ drug interaction $(P=$ $0.02)$ where MLK-SAL and KEF-SAL had the highest values and were significantly different from KEF-DOX and MLK-DOX. Mitral peak flow velocity in SAL was higher $(87.43 \pm 11.38 \mathrm{~ms})$ than DOX $(55.94 \pm 18.11$; $P<0.0001$ ), and MLK-SAL and KEF-SAL had the highest values and KEF-DOX and MLK-DOX had the lowest values $(P=0.004)$. Filling time and ejection time averaged $70.26 \pm 12.56$ and $71.93 \pm 12.28 \mathrm{~ms}$, respectively; however, we found no significant main effects or interactions (Table 3 ).

Fractional shortening in CON was $18.7 \%$ higher than $\operatorname{KEF}(P=0.008)$; CON-SAL values were also significantly higher than KEF-DOX, MLK-DOX, and KEF-SAL $(P=0.04)$. Relative wall thickness was significantly higher in DOX $(0.81 \pm 0.27 \mathrm{~mm})$ compared with SAL $(0.65 \pm 0.16 \mathrm{~mm} ; P=0.006)$. We observed a significant diet effect $(P=0.0003)$ for myocardial performance index, where MLK and KEF were significantly $(32 \%)$ higher than CON (Table 3 ).

\section{DISCUSSION}

The purpose of our study was to examine the effects of kefir and milk-based diets on DOX-induced side effects related to survival, measures of BW, peak grip strength, and heart size and function. Results revealed lower BW in rats after ingesting kefir or milk for $9 \mathrm{wk}$ compared with control-fed animals. These differences persisted at the postinjection time points. Although the kefir-fed animals had large heart masses, cardiac geom-

Table 3. Echocardiographic Doppler blood flow measurements ${ }^{1,2}$

\begin{tabular}{|c|c|c|c|c|c|c|}
\hline Item $^{2}$ & CON-SAL & CON-DOX & MLK-SAL & MLK-DOX & KEF-SAL & KEF-DOX \\
\hline $\mathrm{FT}(\mathrm{ms})$ & $72.54 \pm 11.85^{\mathrm{A}}$ & $74.26 \pm 7.81^{\mathrm{A}}$ & $72.30 \pm 13.65^{\mathrm{A}}$ & $68.59 \pm 15.89^{\mathrm{A}}$ & $69.41 \pm 11.45^{\mathrm{A}}$ & $65.05 \pm 12.95^{\mathrm{A}}$ \\
\hline $\mathrm{ET}(\mathrm{ms})$ & $73.29 \pm 12.69^{\mathrm{A}}$ & $74.76 \pm 8.39^{\mathrm{A}}$ & $75.27 \pm 12.91^{\mathrm{A}}$ & $64.76 \pm 12.37^{\mathrm{A}}$ & $75.23 \pm 12.10^{\mathrm{A}}$ & $68.72 \pm 12.47^{\mathrm{A}}$ \\
\hline Mit. peak $(\mathrm{cm} / \mathrm{s})$ & $82.06 \pm 11.95^{\mathrm{ACD}}$ & $64.72 \pm 9.66^{\mathrm{BDE}}$ & $91.11 \pm 11.86^{\mathrm{AC}}$ & $45.27 \pm 14.03^{\mathrm{BF}}$ & $89.11 \pm 8.90^{\mathrm{AC}}$ & $60.41 \pm 16.21^{\mathrm{BEF}}$ \\
\hline $\mathrm{FS}(\%)$ & $61 \pm 1.10^{\mathrm{AC}}$ & $50 \pm 1.09^{\mathrm{ACD}}$ & $52 \pm 0.63^{\mathrm{ABCD}}$ & $46 \pm 1.49^{\mathrm{ABD}}$ & $43 \pm 0.88^{\mathrm{BD}}$ & $48 \pm 1.03^{\mathrm{BD}}$ \\
\hline RWT (mm) & $0.68 \pm 0.18^{\mathrm{B}}$ & $0.71 \pm 0.21^{\mathrm{A}}$ & $0.63 \pm 0.14^{\mathrm{B}}$ & $0.92 \pm 0.31^{\mathrm{A}}$ & $0.64 \pm 0.17^{\mathrm{B}}$ & $0.77 \pm 0.25^{\mathrm{A}}$ \\
\hline MPI & $0.41 \pm 0.09^{\mathrm{B}}$ & $0.46 \pm 0.14^{\mathrm{B}}$ & $0.54 \pm 0.12^{\mathrm{A}}$ & $0.68 \pm 0.16^{\mathrm{A}}$ & $0.56 \pm 0.17^{\mathrm{A}}$ & $0.53 \pm 0.10^{\mathrm{A}}$ \\
\hline Ao peak $(\mathrm{cm} / \mathrm{s})$ & $81.22 \pm 14.25^{\mathrm{ACD}}$ & $66.03 \pm 22.93^{\mathrm{BDE}}$ & $105.26 \pm 15.35^{\mathrm{AC}}$ & $52.97 \pm 20.30^{\mathrm{BE}}$ & $96.51 \pm 26.75^{\mathrm{AC}}$ & $58.87 \pm 26.70^{\mathrm{BDE}}$ \\
\hline
\end{tabular}

\footnotetext{
${ }_{\mathrm{A}-\mathrm{F}}$ Similar letters indicate no significant difference $(P>0.05)$ among groups.

${ }^{1}$ Data presented as mean $\pm \mathrm{SD}$.

${ }^{2} \mathrm{CON}=$ control; $\mathrm{MLK}=$ milk; $\mathrm{KEF}=$ kefir; $\mathrm{SAL}=$ saline; $\mathrm{DOX}=$ doxorubicin.

${ }^{3} \mathrm{FT}=$ filling time, no significant differences among groups; ET $=$ ejection time, no significant differences among groups; Mit. peak $=$ mitral peak flow velocity, $\operatorname{drug}(P<0.0001)$ and diet $\times \operatorname{drug}$ effect $P=0.004 ; \mathrm{FS}=$ fractional shortening, diet effect $(P=0.008)$ and diet $\times \operatorname{drug}$ effect $(P$ $=0.04) ; \mathrm{RWT}=$ relative wall thickness, $\operatorname{drug}$ effect $P=0.006 ; \mathrm{MPI}=$ myocardial performance index, diet effect $P=0.0003 ;$ Ao peak $=$ aortic peak flow velocity, drug effect $(P<0.0001)$ and diet $\times$ drug interaction $(P=0.02)$.
} 
etry and function data were less clear, and both diets, although not detrimental, did appear to provide added protection against doxorubicin-induced cardiotoxicity.

The use of doxorubicin in the Sprague Dawley rat model is associated with a reduction short-term, postinjection survival (Hayward and Hydock, 2007). These responses vary in magnitude based on the dose of doxorubicin administered and the frequency of injections (a single bolus vs. multiple doses; Hayward and Hydock, 2007). In the current study, a single dose of DOX $(15 \mathrm{mg} / \mathrm{kg})$ resulted in a significant $8 \%$ loss in BW and resulted in a $17 \%$ mortality rate at the 5 -d time point; these results may have achieved significance if the postinjection observation period was longer. Another similar study found that only $40 \%$ of the Sprague Dawley rats receiving a bolus injection of doxorubicin (10 mg/kg i.p.) survived to the 21-d postinjection time point (Hayward and Hydock, 2007). A single $15 \mathrm{mg} / \mathrm{kg}$ dose of DOX is slightly higher than a clinically relevant cumulative dose. This dose was selected for the current study because it represents a dose that induces cardiac dysfunction along with the presence of severe diarrhea, ascites, and high mortality rate, and therefore a greater potential to observe changes related to survival, BW, and cardiac function related to milk or kefir ingestion (Hayward and Hydock, 2007). Components of milk and kefir, including vitamin D and whey protein, may be associated with this protective effect, but research is still developing. An earlier study found that 6 to 8 wk of vitamin $\mathrm{D}\left(1 \alpha, 25\right.$-dihydroxyvitamin $\left.\mathrm{D}_{3}\right)$ supplementation was associated with reduced doxorubicin-induced chromosome damage in the Wister rat bone marrow cells (Kocak et al., 2004). Another study suggested that $25 \mathrm{~d}$ of a whey protein diet attenuated doxorubicin-induced damage, which was linked to an increase in tissue glutathione concentrations (Alexieva et al., 2014). Ours is the first study to explore the use of milk and kefir in the doxorubicin-treated Sprague Dawley rat model.

Although the risk for cancer development and progression was not evaluated in our study, a recent review suggests that milk and milk products are a good source of vitamin D, CLA, butyrate, lactoferrin, and calcium, which may be linked to a reduced risk for the development and progression of cancer (Davoodi et al., 2016). Conversely, other components of milk, including estrogen, contaminants (including pesticides), lactose, and SFA are associated with the development and progression of other cancers (Davoodi et al., 2016). This dichotomy of responses related to dairy product consumption appears to the related to cancer type. For example, in humans, risk for colorectal, liver, bladder, and breast cancer development is reduced with adequate dairy and milk consumption, but dairy and milk consumption appear to be linked to an increase in risk for the develop- ment of prostate and ovarian cancers (Davoodi et al., 2016). Other studies exploring the intake of fermented dairy products, such as cheese, suggest consumption of these products may help protect against the development of cancers, including breast cancer (van't Veer et al., 1989) and ovarian cancer (Faber et al., 2012). These protective effects are hypothesized to be related to the modulation of intestinal microbiota and control of the interplay between immune and epithelial cells (Sánchez et al., 2017).

The milk- and kefir-fed animals weighed significantly less than the control-fed animals at the 9 -wk time point just before doxorubicin or saline injections. Although body composition was not measured in our study, dairy contains casein and whey, which are linked to muscle hypertrophy (Tang et al., 2009), CLA, which may be linked to increased fat oxidation and enhanced appetite regulation (Feltrin et al., 2004; Dougkas et al., 2011), as well as calcium, which may increase fecal fat removal (Christensen et al., 2009). Doxorubicin treatment resulted in a significant $(8 \%)$ reduction in $\mathrm{BW}$ compared with the saline-treated animals, although no significant time by diet interaction was noted; MLKDOX lost an average of $6.6 \%$ of $\mathrm{BW}$, which is almost half of the $11.4 \%$ reduction in BW observed with the CON-DOX animals. It is possible that both MLK and $\mathrm{KEF}$ animals were smaller and may have had less BW to lose; however, their smaller body size did not appear to impair their ability to survive the doxorubicin injection. As mentioned earlier, CON began the study after the milk and kefir dietary interventions were already in progress. This is a noteworthy limitation because the CON animals did not have the opportunity to be randomized to KEF or MLK groups. An exploration into the use of milk and kefir consumption before, during, and after doxorubicin treatment as it relates to favorable, protective changes in body composition has yet to be fully elucidated.

Despite their smaller body sizes, the rodents consuming milk and kefir maintained peak grip strengths that were similar to those of control animals after 9 wk of dietary intervention. Dairy product components, including whey, casein, calcium, and vitamin D, are associated with improved handgrip strength as well as enhanced muscle growth and repair in humans (Hartman et al., 2007; Houston et al., 2007). The use of fermented dairy products to enhance muscle health and function is less clear. A recent study in older adults found that daily ingestion of $210 \mathrm{~g}$ of ricotta cheese for $12 \mathrm{wk}$ resulted in improved appendicular skeletal muscle mass and balance test scores (Alemán-Mateo et al., 2014). As expected, we found a significant drug effect where DOX-treated groups averaged an $11 \%$ lower peak grip strength when compared with saline- 
treated animals (Figure 3). These results are supported by a recently published report that found that a single bolus i.p. injection of doxorubicin $(15 \mathrm{mg} / \mathrm{kg})$ resulted in significantly lower maximal rate of force production and maximal rate of force decline in ex vivo soleus and extensor digitorum longus muscles at $5 \mathrm{~d}$ postinjection (Bredahl et al., 2016). Interestingly, we noted a significant decrease in average peak grip strength in CONDOX and KEF-DOX, but not in MLK-DOX (Figure 3b). It is possible a component in milk is altered in the production in kefir, mitigating this effect; however, relatively little is known about how the kefir production process alters these or other constituents.

Doxorubicin is associated with significant disruption of normal cardiac function. In our study, doxorubicin treatment was associated with $20.8 \%$ smaller heart mass and smaller heart dimension, as measured by septal wall thickness at diastole, LVDd, and posterior wall at systole. Some measures of heart function appeared to follow a similar pattern, with doxorubicin treatment-associated reductions in both mitral peak and aortic peak flow velocities, consistent with another study (Hydock et al., 2009). The milk and kefir diets resulted in animals with smaller body size developing larger hearts with larger measures of LVDs and LVDd when compared with control animals. The effect of the milk and kefir diets on measures of heart function is less clear, with no consistent pattern of diet-related improvements in cardiac function. Aside from the differences in heart size, we made no striking conclusions related to heart geometry or function. Although not clearly evident in our study, it is possible that these dairy products may be may be providing a more profound protective effect elsewhere in the body. Diets high in lower-fat dairy products are associated with improvements in bone health, insulin sensitivity, body composition, and joint health (Rozenberg et al., 2016). It is possible that milk or kefir may be acting to resist the well-established doxorubicin-induced disruptions in skeletal muscle function via managing oxidative stress (Klippel et al., 2016), and potentially acting to preserve the function of myogenic regulatory factors (Quinn and Hydock, 2018). Additionally, both agents may be acting to control immune function and inflammation in the body. A recent study found that the soluble, nonbacterial fraction of kefir enhanced control of chronic inflammation, as demonstrated by decreased tumor necrosis factor $\alpha$ and IL-10 ratio in spontaneously hypertensive rats (Brasil et al., 2018).

In summary, 9 wk of milk or kefir ingestion resulted in lower body size and higher heart mass in Sprague Dawley rats. Additionally, milk preserved peak grip strength after DOX treatment. Aside from a larger heart size in both milk and kefir dietary groups, we observed no consistent protective effects with respect to heart dimensions and function. These findings suggest that long-term milk or kefir ingestion may be helpful in optimizing health before and during doxorubicin treatment. This may be especially important, because many cancer survivors avoid dairy products during chemotherapy and radiation treatment (Osterlund et al., 2004). Kefir contains less lactose, with some varieties claiming to be $99 \%$ lactose-free, which allows individuals with lactose maldigestion to enjoy the benefits of a dairy product. When compared with milk, kefir has also been shown to improve lactose digestion and tolerance in adults (Hertzler and Clancy, 2003). The additional potentially beneficial components of kefir failed to confer health benefits beyond those observed with milk. However, because lactose concentrations are significantly reduced in kefir, this product may be a viable option for cancer patients who are undergoing chemotherapy and want to continue consuming dairy products.

\section{ACKNOWLEDGMENTS}

The authors are grateful to the University of Northern Colorado School of Sport and Exercise Science, the University of Northern Colorado Cancer Rehabilitation Institute, the University of Northern Colorado Sponsored Fellows Program, and the Louisiana State Ag Center for their support of this project.

\section{REFERENCES}

Abu-Elsaad, N. M., A. G. Abd Elhameed, A. El-Karef, and T. M. Ibrahim. 2015. Yogurt containing the probacteria Lactobacillus acidophilus combined with natural antioxidants mitigates doxorubicin-induced cardiomyopathy in rats. J. Med. Food 18:950-959.

ACS (American Cancer Society). 2017. Cancer Statistics Fact Sheet. Accessed May 1, 2018. https://www.cancer.org/research/cancer -facts-statistics/all-cancer-facts-figures/cancer-facts-figures-2017 .html.

Ahmed, Z., Y. Wang, A. Ahmad, S. T. Khan, M. Nisa, H. Ahmad, and A. Afreen. 2013. Kefir and health: A contemporary perspective. Crit. Rev. Food Sci. Nutr. 53:422-434.

Alemán-Mateo, H., V. R. Carreon, L. Macias, H. Astiazaran-Garcia, A. C. Gallegos-Aguilar, and J. R. Enriquez. 2014. Nutrient-rich dairy proteins improve appendicular skeletal muscle mass and physical performance, and attenuate the loss of muscle strength in older men and women subjects: A single-blind randomized clinical trial. Clin. Interv. Aging 9:1517-1525.

Alexieva, B., S. Stoitsova, V. Pavlova, T. Paunova, and E. Nikolova. 2014. Whey protein concentrate as a protective agent against doxorubicin-induced cardiotoxicity in mice. Biotechnol. Biotechnol. Equip. 23(Suppl. 1):493-497.

Brasil, G. A., M. A. Silva-Cutini, F. S. A. Moraes, T. M. C. Pereira, E. C. Vasquez, D. Lenz, N. S. Bissoli, D. C. Endringer, E. M. de Lima, V. C. Biancardi, J. F. Maia, and T. U. de Andrade. 2018. The benefits of soluble non-bacterial fraction of kefir on blood pressure and cardiac hypertrophy in hypertensive rats are mediated by an increase in baroreflex sensitivity and decrease in angiotensin-converting enzyme activity. Nutrition 51-52:66-72. 
Bredahl, E. C., K. B. Pfannenstiel, C. J. Quinn, R. Hayward, and D. S. Hydock. 2016. Effects of exercise on doxorubicin-induced skeletal muscle dysfunction. Med. Sci. Sports Exerc. 48:1468-1473.

Chatterjee, K., J. Zhang, N. Honbo, and J. S. Karliner. 2010. Doxorubicin cardiomyopathy. Cardiology 115:155-162.

Christensen, R., J. K. Lorenzen, C. R. Svith, E. M. Bartels, E. L. Melanson, W. H. Saris, A. Tremblay, and A. Astrup. 2009. Effect of calcium from dairy and dietary supplements on faecal fat excretion: a meta-analysis of randomized controlled trials. Obes. Rev. 10:475-486.

Davoodi, S. H., R. Shahbazi, S. Esmaeili, S. Sohrabvandi, A. Mortazavian, S. Jazayeri, and A. Taslimi. 2016. Health-related aspects of milk proteins. Iran. J. Pharm. Res. 15:573-591.

Desai, V. G., E. H. Herman, C. L. Moland, W. S. Branham, S. M. Lewis, K. J. Davis, N. I. George, T. Lee, S. Kerr, and J. C. Fuscoe. 2013. Development of doxorubicin-induced chronic cardiotoxicity in the B6C3F1 mouse model. Toxicol. Appl. Pharmacol. 266:109 121.

Dougkas, A., C. K. Reynolds, I. D. Givens, P. C. Elwood, and A. M. Minihane. 2011. Associations between dairy consumption and body weight: a review of the evidence and underlying mechanisms. Nutr. Res. Rev. 24:72-95.

Faber, M. T., A. Jensen, M. Sogaard, E. Hogdall, C. Hogdall, J. Blaakaer, and S. K. Kjaer. 2012. Use of dairy products, lactose, and calcium and risk of ovarian cancer-Results from a Danish case-control study. Acta Oncol. 51:454-464.

Farnworth, E. 2015. Kefir: From folklore to reglatory approval. J. Nutraceuticals Funct. Med. Foods 1:48-68. https://doi.org/10.1300/ J133v01n04_05.

Feltrin, K. L., T. J. Little, J. H. Meyer, M. Horowitz, A. J. Smout, J. Wishart, A. N. Pilichiewicz, T. Rades, I. M. Chapman, and C. Feinle-Bisset. 2004. Effects of intraduodenal fatty acids on appetite, antropyloroduodenal motility, and plasma CCK and GLP-1 in humans vary with their chain length. Am. J. Physiol. Regul. Integr. Comp. Physiol. 287:R524-R533.

Garofalo, C., A. Osimani, V. Milanovic, L. Aquilanti, F. De Filippis, G. Stellato, S. Di Mauro, B. Turchetti, P. Buzzini, D. Ercolini, and F. Clementi. 2015. Bacteria and yeast microbiota in milk kefir grains from different Italian regions. Food Microbiol. 49:123-133.

Hartman, J. W., J. E. Tang, S. B. Wilkinson, M. A. Tarnopolsky, R. L. Lawrence, A. V. Fullerton, and S. M. Phillips. 2007. Consumption of fat-free fluid milk after resistance exercise promotes greater lean mass accretion than does consumption of soy or carbohydrate in young, novice, male weightlifters. Am. J. Clin. Nutr. 86:373-381.

Hayward, R., and D. S. Hydock. 2007. Doxorubicin cardiotoxicity in the rat: An in vivo characterization. J. Am. Assoc. Lab. Anim. Sci. 46:20-32.

Hertzler, S. R., and S. M. Clancy. 2003. Kefir improves lactose digestion and tolerance in adults with lactose maldigestion. J. Am. Diet. Assoc. 103:582-587.

Houston, D. K., M. Cesari, L. Ferrucci, A. Cherubini, D. Maggio, B. Bartali, M. A. Johnson, G. G. Schwartz, and S. B. Kritchevsky. 2007. Association between vitamin D status and physical performance: The InCHIANTI study. J. Gerontol. A Biol. Sci. Med. Sci. 62:440-446.
Hydock, D. S., C. Y. Lien, and R. Hayward. 2009. Anandamide preserves cardiac function and geometry in an acute doxorubicin cardiotoxicity rat model. J. Cardiovasc. Pharmacol. Ther. 14:59-67.

Klippel, B. F., L. B. Duemke, M. A. Leal, A. G. Friques, E. M. Dantas, R. F. Dalvi, A. L. Gava, T. M. Pereira, T. U. Andrade, S. S. Meyrelles, B. P. Campagnaro, and E. C. Vasquez. 2016. Effects of kefir on the cardiac autonomic tones and baroreflex sensitivity in spontaneously hypertensive rats. Front. Physiol. 7:211.

Kocak, N., H. Ustun, M. D. Gulkac, A. O. Kanli, A. Borazan, and A. Yilmaz. 2004. Effects of 10alpha,25-dihydroxyvitamin D3 on doxorubicin-induced chromosomal aberrations in rat bone marrow cells. Acta Oncol. 43:204-208.

Langer, S. W. 2014. Dexrazoxane for the treatment of chemotherapyrelated side effects. Cancer Manag. Res. 6:357-363.

Osterlund, P., T. Ruotsalainen, K. Peuhkuri, R. Korpela, A. Ollus, M. Ikonen, H. Joensuu, and I. Elomaa. 2004. Lactose intolerance associated with adjuvant 5-fluorouracil-based chemotherapy for colorectal cancer. Clin. Gastroenterol. Hepatol. 2:696-703.

Piotrowska, I., M. Isalan, and M. Mielcarek. 2017. Early transcriptional alteration of histone deacetylases in a murine model of doxorubicin-induced cardiomyopathy. PLoS One 12:e0180571.

Pugazhendhi, A., T. Edison, B. K. Velmurugan, J. A. Jacob, and I. Karuppusamy. 2018. Toxicity of doxorubicin (Dox) to different experimental organ systems. Life Sci. 200:26-30.

Quinn, C. J., and D. S. Hydock. 2018. Effects of endurance exercise and doxorubicin on skeletal muscle myogenic regulatory factor expression. Muscles Ligaments Tendons J. 7:418-425.

Rozenberg, S., J. J. Body, O. Bruyere, P. Bergmann, M. L. Brandi, C. Cooper, J. P. Devogelaer, E. Gielen, S. Goemaere, J. M. Kaufman, R. Rizzoli, and J. Y. Reginster. 2016. Effects of dairy products consumption on health: Benefits and beliefs - A commentary from the Belgian Bone Club and the European Society for Clinical and Economic Aspects of Osteoporosis, Osteoarthritis and Musculoskeletal Diseases. Calcif. Tissue Int. 98:1-17.

Sánchez, B., S. Delgado, A. Blanco-Miguez, A. Lourenco, M. Gueimonde, and A. Margolles. 2017. Probiotics, gut microbiota, and their influence on host health and disease. Mol. Nutr. Food Res. $61: 1600240$.

Sharma, S., R. L. Singh, and P. Kakkar. 2011. Modulation of Bax/Bcl2 and caspases by probiotics during acetaminophen induced apoptosis in primary hepatocytes. Food Chem. Toxicol. 49:770-779.

Tang, J. E., D. R. Moore, G. W. Kujbida, M. A. Tarnopolsky, and S. M. Phillips. 2009. Ingestion of whey hydrolysate, casein, or soy protein isolate: Effects on mixed muscle protein synthesis at rest and following resistance exercise in young men. J. Appl. Physiol. 107:987-992

van't Veer, P., J. M. Dekker, J. W. Lamers, F. J. Kok, E. G. Schouten, H. A. Brants, F. Sturmans, and R. J. Hermus. 1989. Consumption of fermented milk products and breast cancer: A case-control study in The Netherlands. Cancer Res. 49:4020-4023.

Yang, F., S. S. Teves, C. J. Kemp, and S. Henikoff. 2014. Doxorubicin, DNA torsion, and chromatin dynamics. Biochim. Biophys. Acta 1845:84-89. 